\title{
The relative contribution of urine extravasation to elevate plasma creatinine levels in acute unilateral ureteral obstruction
}

\author{
Barak Rosenzweig, MD; Jehonathan H. Pinthus, MD, FRCSC; ${ }_{;}^{*}$ Nir Kleinmann, MD; Erel Joffe, MD; \\ Tomer Erlich, MD; ${ }^{*}$ Eddie Fridman, MD; ${ }^{*}$ Harry Winkler, MD;* Yoram Mor, MD; Jacob Ramon, MD;* \\ Zohar A. Dotan, MD*
}

*Department of Urology, The Chaim Sheba Medical Center, Tel Hashomer, Ramat-Gan, Sackler School of Medicine, Tel-Aviv University, Israel; †Department of Surgery, Division of Urology, McMaster University, Hamilton, ON; §School of Biomedical Informatics, University of Texas Health Science Center, Houston, TX; *Department of Pathology, The Chaim Sheba Medical Center, Tel Hashomer, Ramat-Gan, Sackler School of Medicine, Tel-Aviv University, Israel

Cite as: Can Urol Assoc J 2015;9(7-8):E428-33. http://dx.doi.org/10.5489/cuai.2804 Published online July 17, 2015.

\section{Abstract}

Introduction: Rising levels of plasma creatinine in the setting of acute unilateral ureteral obstruction (AUUO) often reflects acute renal failure, mandating kidney drainage. We hypothesize that reabsorption of peri-renal urine extravasation (PUE), a common result of UUO, contributes significantly to the elevation in plasma creatinine, rendering the latter an inaccurate benchmark for renal function. We explored this hypothesis in a rat model of AUUO and PUE. Methods: In total, 20 rats were equally divided into 4 groups. Groups 1 and 2 underwent unilateral ligation of the ureter with infiltration of rat's urine (index group) or saline (control) into the peri-renal space. Two additional control groups underwent perirenal injection of either urine or saline without AUUO. Plasma creatinine levels were determined immediately prior to the procedure (T0), and hourly for 3 hours (T1, T2 and T3). Renal histology was investigated after 3 hours.

Results: Rats in the index group had a significantly greater increase in plasma creatinine levels over 3 hours compared to all other groups $(p<0.05)$. At T3, average plasma creatinine levels for the index group increased by $96 \%(0.49 \pm 0.18 \mathrm{mg} / \mathrm{dL})$ compared to $46 \%(0.23 \pm 0.06 \mathrm{mg} / \mathrm{dL}$ increase $)$ in the AUUO and saline group, and less than $15 \%$ rise in both the non-obstructed control groups. Our study limitations includes lack of spontaneous PUE and intraperitoneal surgical approach.

Conclusions: Absorption of peri-renal urine in the presence of AUUO is a significant contributor to rising plasma creatinine levels, beyond those attributable to the obstruction alone, and may overestimate the extent of the true renal functional impairment.

\section{Introduction}

Acute unilateral ureteral obstruction (AUUO) is a clinical scenario commonly encountered due to obstructing stone, trauma, malignancy, and endometriosis., ${ }^{1,2}$ AUUO is often accompanied by a rise in plasma creatinine levels, which raises the concern for acute renal failure secondary to the obstruction, for which urgent kidney drainage is often recommended. ${ }^{3}$ As high as $18 \%$ of patients with acute ureteral obstruction also present with peri-renal urine extravasation (PUE). 4,5 Moreover, peri-renal edema and tissue stranding were reported in up to $65 \%$ of renal colic imagings due to obstructing stones. ${ }^{6}$ Several previous studies have inconsistently described urine extravasation as either a good prognostic sign for spontaneous stone passage, shorter hospitalization and fewer subsequent therapeutic procedures, or associated with delayed diagnosis, infection and renal failure in the clinical setting of ureteral obstruction..$^{5,7}$

Peri-pelvic urine extravasation usually results from a rupture of the calyceal fornix secondary to high intra-pelvic pressures. ${ }^{8,9}$ The extravasated urine is subsequently re-absorbed by lymphatic or venous channels. ${ }^{8}$ Urine extravasation has been suggested to correlate with rising serum creatinine levels in a well-functioning transplanted kidney. ${ }^{10}$ However, to the best of our knowledge, there are no reports of studies investigating the relative contribution of extravasated urine re-absorption to the overall increase of serum creatinine levels following AUUO. We believe that re-absorption of extravasated urine is a major contributor to the elevation in serum creatinine in cases of AUUO.

\section{Methods}

\section{Experimental design}

This study was carried out in strict accordance with the recommendations in the guidelines of the standing committee on animal research (permit number: 0962/08). Eight weekold male Wistar rats, with an average weight of $300 \mathrm{~g}$, were used. Rats were housed in regular cages for an acclimation 
period of 5 days, situated in an animal room temperature of $22^{\circ} \mathrm{C}$ with a 12 -hour light $/ 12$-hour dark cycle. Rats were maintained on standard rat chow diet and given tap water to drink ad libitum. Prior to surgery, rats were held in metabolic cages and 15-hour urine samples were collected.

Under sedation with isoflurane inhalation, all rats were subsequently anesthetized with intraperitoneal (IP) ketamine $(100 \mathrm{mg} / \mathrm{kg})$ plus xylazine $(15 \mathrm{mg} / \mathrm{kg})$ after being preoperatively hydrated by subcutaneous injection of 5 cc saline mixed with indigo carmine (5,5'-indigodisulfonic acid sodium salt, $0.37 \mathrm{mg} / \mathrm{L}$ ). Additional doses of ketamine/xylazine anesthesia were given to maintain proper anesthesia throughout the entire procedure. The left kidney and ureter were approached using a left subcostal intraperitoneal incision. Leica M690 microscope with $\times 10$ magnification was used for the dissection.

In total, 20 rats were equally divided into 4 groups (Fig. 1). The index group underwent acute distal left ureteral obstruction as previously described (T0). A volume of $0.5 \mathrm{cc}$ of each individual rat's urine (equivalent to the urinary output of a renal unit of nearly 2 hours according to urine output measurement in the preoperative metabolic cage) was dyed by adding $0.05 \mathrm{cc}$ of indigo carmine $(0.37 \mathrm{mg} / \mathrm{L})$ and infiltrated into the left peri-renal retroperitoneal tissue using a 30-gauge needle. White cotton wool was immediately applied to the injection site to exclude urine leakage through the needle entry point into the peritoneal cavity. Three groups served as controls: the first control group underwent the exact same protocol except for being injected with indigo carmine-dyed saline $(0.37 \mathrm{mg} / \mathrm{L})$ instead of urine. Two additional control groups were injected with either saline or urine with no ureteral obstruction performed. Baseline blood analyses for plasma creatinine were taken from left jugular vein (25-gauge needle, $0.5 \mathrm{~mL}$ ) of all rats prior to ureteral obstruction and injection (T0). Three additional blood samples were taken hourly using the same technique (T1, T2 and T3). Rats were sacrificed at T3 by Isoflurane asphyxiation and exsanguination. Post-mortem dissection confirmed ureteral obstruction (when performed), absence of traumatic kidney injury, and the presence of perinephric traces of injected urine or saline. Sampled histological assessment was performed for one animal in each group (8 kidney units), using hematoxylin and eosin (H\&E) and periodic acid-Schiff (PAS) stains.

To verify that our experimental model of PUE was wellcontrolled and our readings were not influenced by spontaneous urinary extravasation induced by the acute surgical obstruction of the ureter, we conducted the following experiment: Four rats underwent meticulous dissection of the left kidney isolating it with its intact renal vasculature from all adherent peri-renal tissue. The isolated kidney was then engulfed by white cotton wool in an attempt to identify any indigo carmine urine extravasation. Four additional rats that did not undergo peri-renal dissection served as controls. In both groups the left ureter was ligated (Vicryl 3-0) and transected to create a complete obstruction (T0). All rats were aggressively hydrated with hourly subcutaneous injections of 5 cc saline mixed with indigo carmine (5,5'-indigodisulfonic acid sodium salt, $0.37 \mathrm{mg} / \mathrm{L}$ ). Prior to ureteral obstruction, a blood sample for plasma creatinine baseline value (T0) was withdrawn from the left jugular vein (25-gauge needle, $0.5 \mathrm{~mL}$ ). Three additional blood samples were taken hourly using the same technique (T1, T2 and T3).

Aggressive hydration was performed in both experiments to prevent pre-renal renal failure due to prolonged laparotomy, neck incision and recurrent blood analysis. Serum creatinine was measured at our institute's automatic laboratory using the Jaffe reaction principle. We used the repeated analyses of variance (ANOVA) test to compare plasma creatinine values between the individual rats and between the 4 groups.

\section{Results}

The mean basal urine output measured in our model was $0.61 \mathrm{~mL} / \mathrm{h}$ (median 0.56 , range: $0.2-1.4 \mathrm{~mL} / \mathrm{h}$, standard deviation $0.3 \mathrm{~mL}$ ). Accordingly, the mean baseline urine output per kidney unit was estimated at $0.31 \mathrm{~mL} / \mathrm{h}$ (median 0.28 $\mathrm{ml} / \mathrm{h}$, range: $0.1-0.7 \mathrm{~mL} / \mathrm{h}$, standard deviation $0.15 \mathrm{~mL}$ ). The induction of AUUO produced a significant rise in serum creatinine levels (Fig. 2), but did not cause any spontaneous extravasation of urine as demonstrated by a lack of dyed urine on the cotton wool engulfing the dissected kidney. When we simulated PUE by infiltrating urine around the left kidney as previously described (Fig. 3), the index

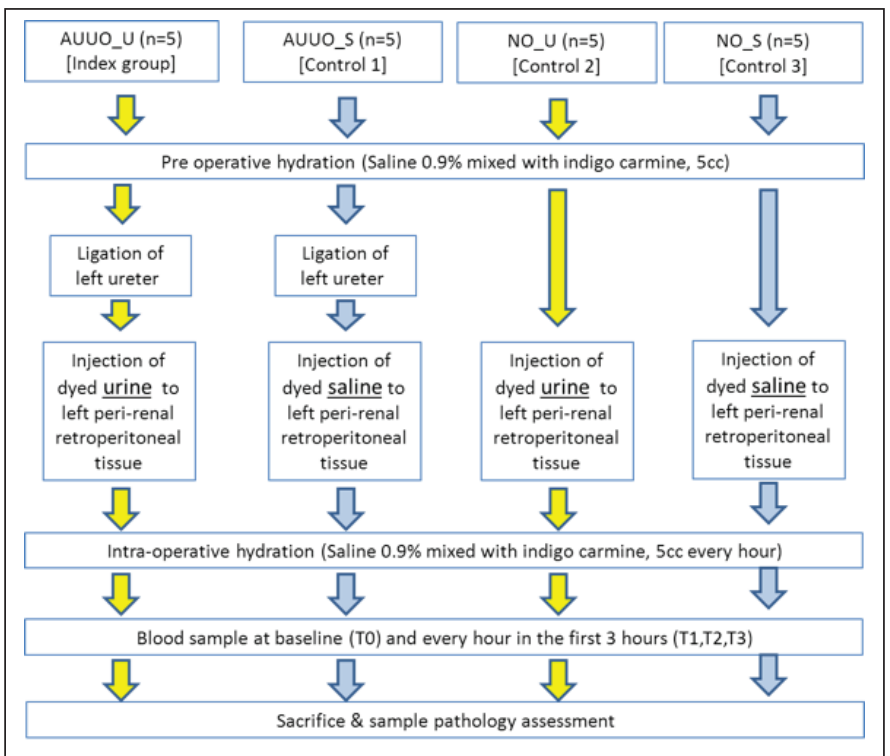

Fig. 1. Study design. AUUO: acute unilateral ureteral obstruction; U: retroperitoneal urine injection; $\mathrm{S}$ : retroperitoneal saline injection; NO: non-obstructed ureter. 


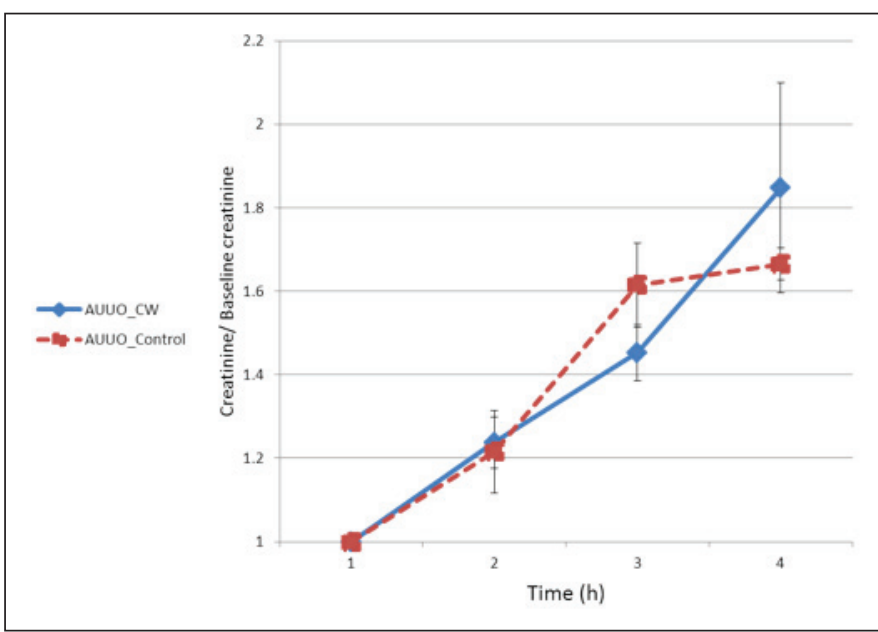

Fig. 2. Change in plasma creatinine over time. AUUO: acute unilateral ureteral obstruction; CW: cotton wool, engulfing the dissected kidney. Standard error represented by error lines

group had a significantly higher increase in creatinine over time compared to all the control groups $(p<0.05)$. At 3 hours, the average creatinine for the index group increased by $96 \%(0.49 \pm 0.18 \mathrm{mg} / \mathrm{dL}$ increase) compared to $46 \%$ $(0.23 \pm 0.06 \mathrm{mg} / \mathrm{dL}$ increase $)$ in the AUUO plus the saline group, and less than $15 \%$ rise in both non-obstructed control groups (Fig. 4).

Accordingly, the average rate of increase in creatinine was at least twice as high for the index group compared to the controls $(0.164 \mathrm{mg} / \mathrm{dL} / \mathrm{h}$ to $0.078 \mathrm{mg} / \mathrm{dL} / \mathrm{h}$ or less $)$. Post-mortem dissection ruled out traumatic kidney injury in all groups. Histological assessment identified signs of obstructive uropathy in the kidneys with ligated ureters, as opposed to the non-obstructed kidneys. The obstructed kidney showed hydronephrosis and prominent dilatation of non-proximal tubules, with relative reactive thinning of the lining cuboidal epithelium (Fig. 5a). Intraglomerular Bowman's capsule dilatation and edema were noted without evidence of glomerular and/or epithelial cell damage in the proximal tubules (Fig. 5b).

\section{Discussion}

We evaluated the effect of peri-renal urine infiltration, as a surrogate to urine extravasation, on the elevation of plasma creatinine.

Accurate assessment of the extent of kidney damage is pertinent to the clinical management of a patient with an acute obstruction and presumed renal failure. A commonly used clinical estimation of renal function is based upon serum creatinine level as a surrogate marker, applying the Cockcroft-Gault formula. ${ }^{11}$ Accordingly, elevated creatinine is considered a marker of renal failure in the setting of ureteral obstruction and often indicates the ability of the affected renal unit(s) to drain urine properly. ${ }^{3}$ Therefore, there is a

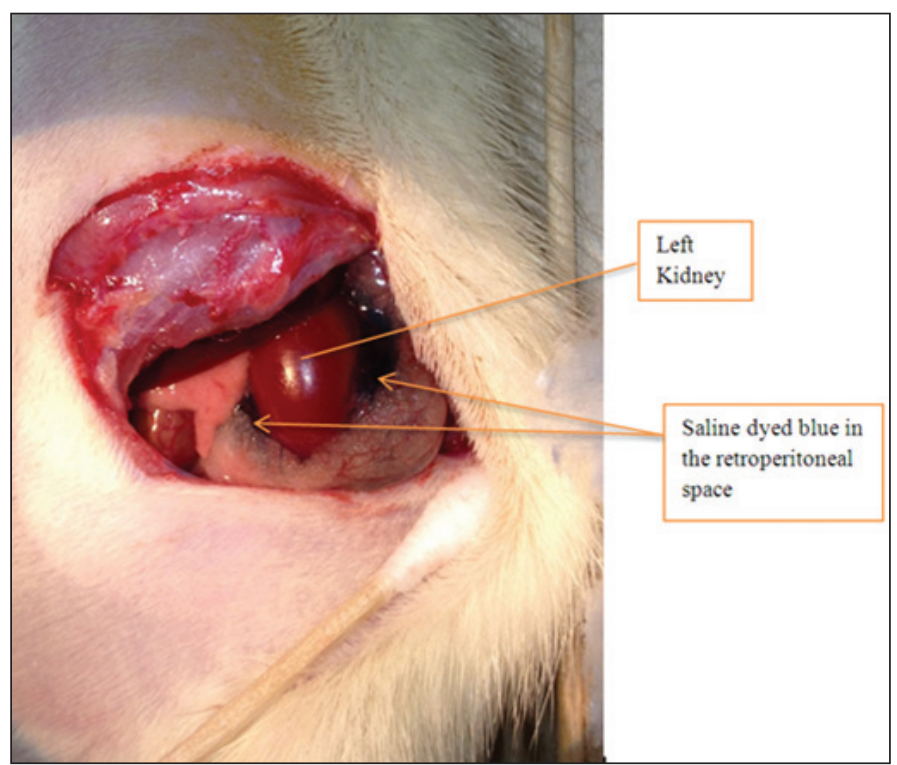

Fig. 3. Subcostal transperitoneal incision showing rat's left kidney with indigo carmine-dyed saline injected into peri-hilar and lateral retroperitoneal cavities.

strong case for investigating the exact mechanism responsible for plasma creatinine elevation in the setting of AUUO.

Hinman identified high peri-pelvic and peri-ureteral concentrations of preoperatively administered Pyridium in the setting of iatrogenic ureteral obstruction. ${ }^{9}$ The suggested mechanism for the urine extravasation includes rupture of the calyceal fornix secondary to rapidly increasing intrapelvic pressures, with extravasated urine being re-absorbed

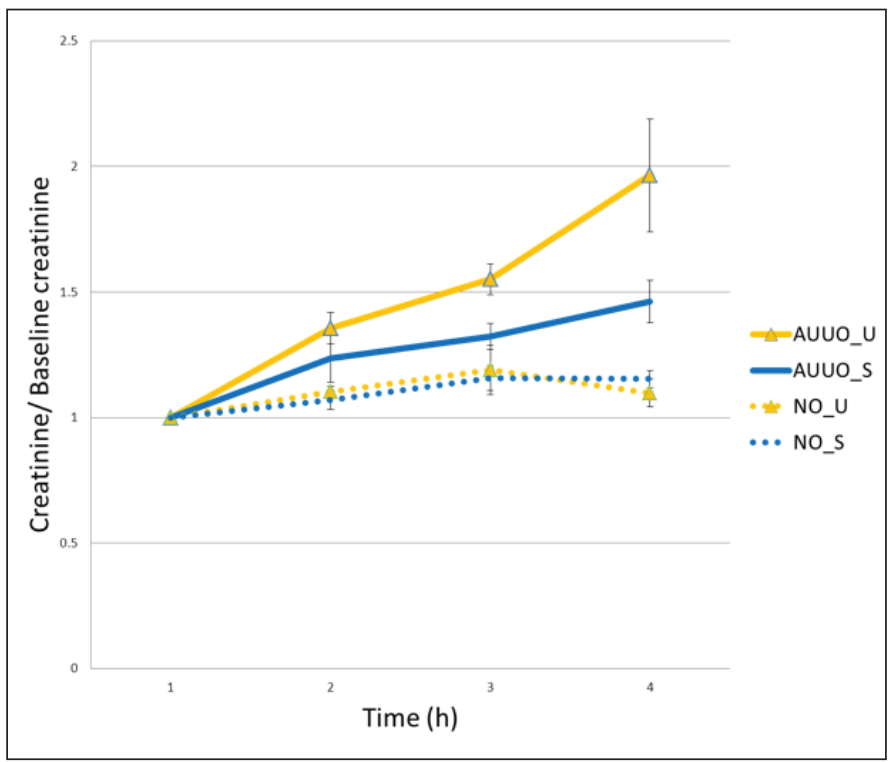

Fig. 4. Change in plasma creatinine over time exemplifying higher creatinine values for obstructed kidneys with retroperitoneal urine injection. AUUO: acute unilateral ureteral obstruction; U: retroperitoneal urine injection; S: retroperitoneal saline injection; NO: non-obstructed ureter. Standard error represented by error lines. 


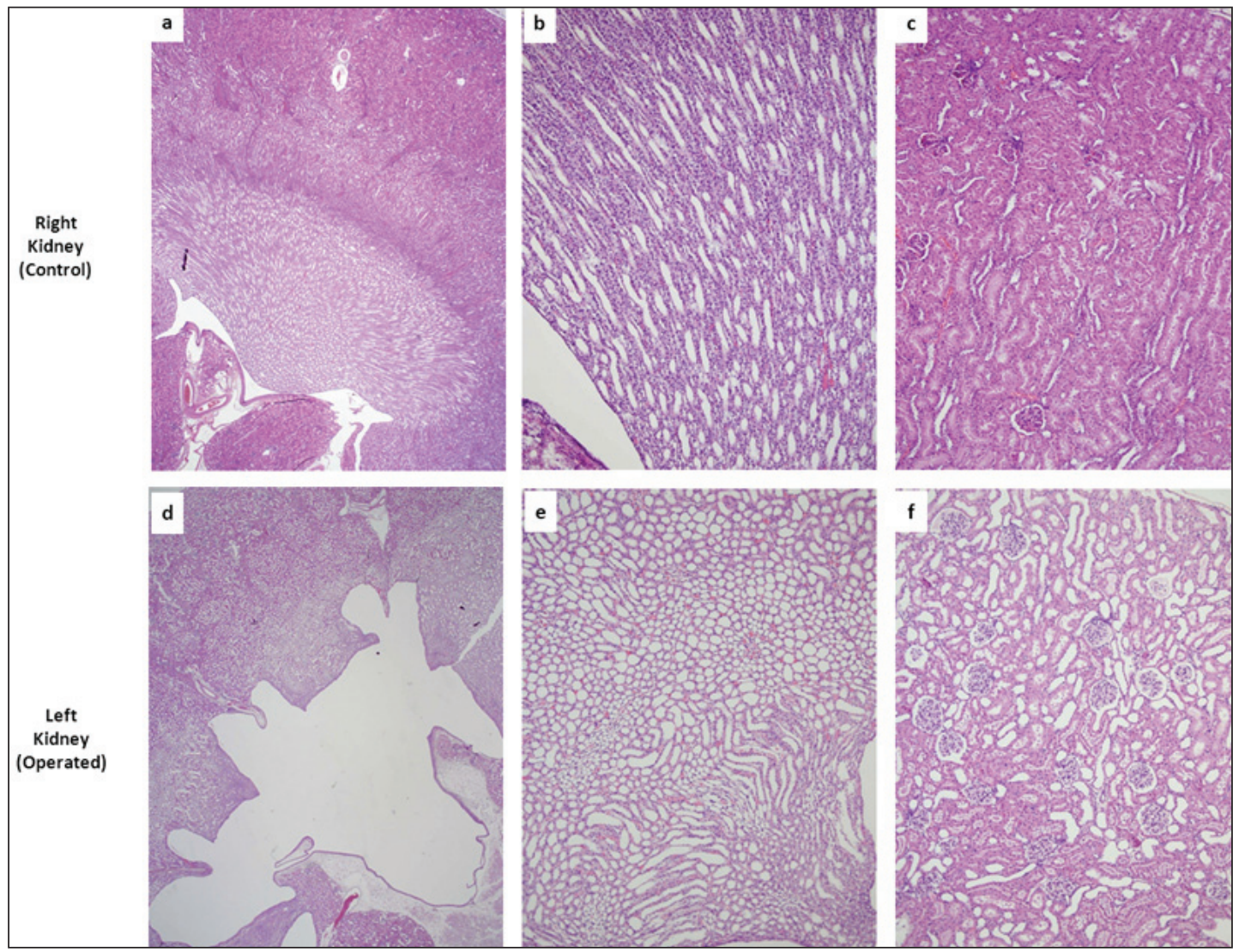

Fig. 5a. Examples of pathologic changes in the operated as opposed to control kidneys: D: hydronephrosis and prominent dilatation of non-proximal tubules with; $E$ and $F$ : relative reactive thinning of the lining cuboidal epithelium; $A$ and $D$ : hematoxylin \& eosin $(H \& E) \times 20 ; B$ and $E: H \& E \times 100$ medulla; $C$ and $F: H \& E \times 100$ cortex.

by either the venous or lymphatic routes. Earlier reports of increased rates of thoracic duct flow and radio-isotope appearance in the thoracic duct following ureteral obstruction further support the re-absorption concept. ${ }^{12,13}$

To the best of our knowledge, no rat model of urine extravasation in the setting of AUUO has been previously described. Our model has not demonstrated visible spontaneous PUE following ureteral obstruction. Yet, we did show an increase in plasma creatinine levels over time, indicating that genuine renal functional impairment does occur in AUUO without PUE. Exemplifying no spontaneous PUE, we have developed a model which artificially simulates PUE by direct urine infiltration around the dissected obstructed kidney. The increase in plasma creatinine levels in our rat model was significantly higher in the index group (AUUO and PUE), compared to all control groups $(p<0.05)$ (Fig. 4). Post-mortem dissection showed clear evidence of fluid traces at the injected pockets with no evidence of traumatic kidney injury, confirming that the injected fluids were largely absorbed and ruling out traumatic kidney injury as a possible alternative explanation for the rise in plasma creatinine. The histological assessment validated induction of obstructive uropathy by showing signs of acute kidney injury in the obstructed kidneys.
The results of the current study are in accordance with previous studies, and suggest that the acute rise in plasma creatinine in the setting of acute kidney obstruction and urine extravasation can be partly attributed to urinary absorption and does not really reflect pure renal failure. Indeed, such pseudo renal failure due to urinary re-absorption has been previously described, in the setting of intra-peritoneal urine leak and anecdotally, due to retroperitoneal urinary leakage after kidney transplantation. ${ }^{10,14-16}$ Our model further elaborated and investigated a similar concept, and suggested that absorption of PUE on top of AUUO is a major contributor in the rise in plasma creatinine levels. Plasma creatinine rise, despite a normal functioning contralateral kidney, can be explained by several mechanisms: (1) creatinine overload which overcomes the filtration power of that non-obstructed kidney; and (2) reno-renal communication as exemplified by the reno-renal reflex, such mechanism causes a contralateral kidney inhibitory effect with no glomerular filtration rate rise in the rat model. ${ }^{17}$ Lack of obstruction and these suggested mechanisms, along with aggressive hydration and hyper-elimination of two functional kidneys, explain why no plasma creatinine rise was seen in the two non-obstructed groups (Fig. 4). 

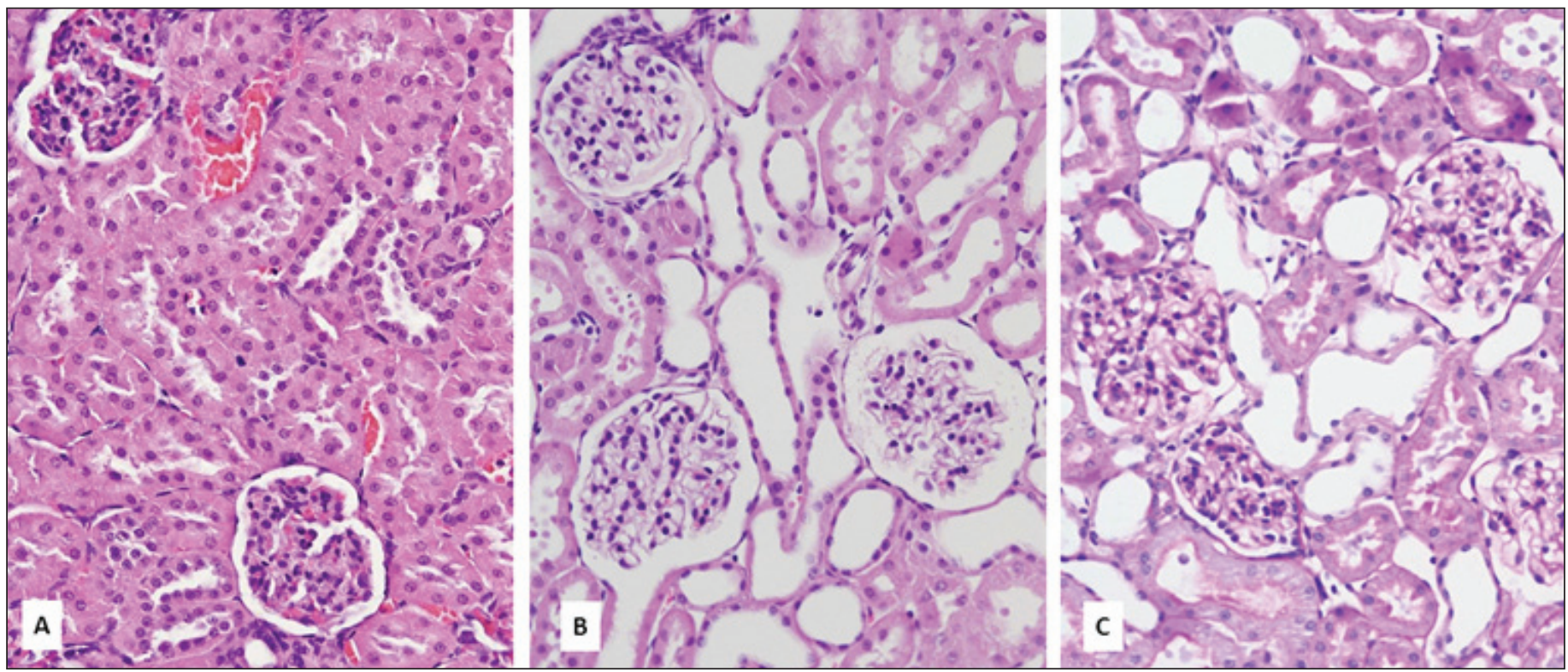

Fig. 5b. Examples of pathologic changes in the operated as opposed to control kidneys. A: non-obstructed right kidney- control. Hematoxylin and eosin (H\&E) $\times 400$. B and C: obstructed, operated left kidney, H\&E and periodic acid-Schiff $\times 400$, respectively. Prominent dilatation of non-proximal tubules with relative reactive thinning of the lining cuboidal epithelium. Intraglomerular Bowman's capsule dilatation and edema are noted without evidence of glomerular and/or epithelial cell damage in proximal tubules.

Our study has several limitations. First, we were unable to produce visible spontaneous extravasation of urine following unilateral ureteral obstruction. A possible explanation is that the study rats did not have significant elevated intra-pelvic pressure, to induce "pop-off" of the urinary system and cause extravasation. Spontaneous human urinary extravasation was correlated with absolute pressure in the urinary collecting system, as well as with the pressure increase rate, the latter serving as the more important factor of the two. ${ }^{18,19} \mathrm{~A}$ second limitation is the use of the transperitoneal approach. Meticulous surgical technique was applied to avoid possible peritoneal urine absorption, including deep injection with small diameter needle, high magnification microscope, and white cotton wool application to rule out leak of dyed urine. Post-mortem dissection showed clear evidence of retro-peritoneal fluid traces at the injected pockets.

Despite the intuitive correlation between retroperitoneal urine extravasation in the setting of $A \cup \cup O$ and spurious renal failure, research and clinical data are scarce. Our data suggest that increased serum creatinine levels might be a non-accurate measurement tool of renal functional impairment in the setting of AUUO and PUE which might lead to unnecessary drainage of the affected kidney. Being familiar with the consequences and the potential complications of such drainage and its effect on patients' quality of life, ${ }^{20}$ we believe further research should take place to elaborate our understanding of PUE.

\section{Conclusion}

The presence of urine in the peri-renal space in the AUUO rat model is associated with increased plasma creatinine levels beyond those attributable to the obstruction alone. Our findings suggest that measurements of plasma creatinine in such a setting may therefore overestimate the extent of the true renal functional impairment. Further research is needed to investigate the clinical implication of our findings.

Competing interests: The authors declare no competing financial or personal interests.

This paper has been peer-reviewed.

\section{Reference}

1. Picozzi SCM, Marenghi C, Casellato S, et al. Management of ureteral calculi and medical expulsive therapy in emergency departments. J Emerg Trauma Shock 2011;4:70-6. http://dx.doi.org/10.4103/09742700.76840

2. Singh I, Strandhoy JW, Assimos DG. Campbell-Walsh Urology. 10th ed. Wein AJ, Kavoussi LR, Novick AC, Partin AW, Peters CA, editors. Philadelphia, PA: Elsevier Saunders; 2012:1087-8.

3. Nakada SY, Hsu THS. Campbell-Walsh Urology. 10th ed. Wein AJ, Kavoussi LR, Novick AC, Partin AW, Peters CA, editors. Philadelphia, PA: Elsevier Saunders; 2012:1163-4.

4. Kosehan D, Akin K, Topcu A, et al. Spontaneous urinary extravasation: Detection rate with 64-row multidetector computed tomography in patients presenting with acute abdomen. Emerg Radiol 2013;20:273-7. http://dx.doi.org/10.1007/s10140-013-1119-7

5. Chapman JP, Gonzalez J, Diokno AC. Significance of urinary extravasation during renal colic. Urology 1987;30:541-5. http://dx.doi.org/10.1016/0090-4295(87)90432-8 
Urine extravasation in acute unilateral ureteral obstruction

6. Katz DS, Lane MJ, Sommer FG. Unenhanced helical CT of ureteral stones: Incidence of associated urinary tract findings. AJR Am J Roentgenol 1996;166:1319-22. http://dx.doi.org/10.2214/ air. 166.6 .8633440

7. Kettlewell $M$, Walker $M$, Dudley $N$, et al. Spontaneous extravasation of urine secondary to ureteric obstruction. Br J Urol 1973;45:8-14. http://dx.doi.org/10.1111/i.1464-410X.1973.tb00038.x

8. Hinman F. Peripelvic extravasation during intravenous urography, evidence for an additional route for backflow after ureteral obstruction. J Urol 1961;85:385-95.

9. Harrow BR, Sloane JA. Pyelorenal extravasation during excretory urography. J Urol 1961;85:995-1005.

10. Schultze Kool L, Blickman JG, van Es A, et al. Retroperitoneal reabsorption of extravasated urine in renal transplant patients. Radiology 1984;153:625-6. http://dx.doi.org/10.1148/radiology.153.3.6387786

11. Cockcroft DW, Gault MH. Prediction of creatinine clearance from serum creatinine. Nephron 1976;16:3141. http://dx.doi.org/10.1159/000180580

12. Chisholm GD, Rivero OR, Calnan JS. The permeability of the renal pelvis during ureteric obstruction. $\mathrm{Br} J$ Surg 1967;54:1023-6. http://dx.doi.org/10.1002/bjs. 1800541212

13. Myint MK, Murphy JJ. The renal lymphatics. I. The effect of diuresis and acute ureteral obstruction upon the rate of flow and composition of thoracic duct lymph. Surg Forum 1957;7:656-60.

14. Chow KM, Lam CM, Szeto CC. Pseudo-renal failure following total abdominal hysterectomy. J Nephrol 2005;18:442-6.
15. Heyns $\mathrm{CF}$, Rimington PD. Intraperitoneal rupture of the bladder causing the biochemical features of renal failure. Br J Urol 1987;60:217-22. http://dx.doi.org/10.1111/i.1464-410X.1987.tb05486.x

16. Dees $A$, Kluchert $S A$, van Vliet AC. Pseudo-renal failure associated with internal leakage of urine. Neth J Med 1990;37:197-201.

17. Kopp UC, Olson LA, DiBona GF. Renorenal reflex responses to mechano- and chemoreceptor stimulation in the dog and rat. Am J Physiol 1984;246:F67-77.

18. Kohler R. Investigations on backflow in retrograde pyelography: A roentgenological and clinical study. Acta Radiol 1953;39:1-92.

19. Kiil F. Pressure resordings and radiograophic studies of the normal and diseased urinary tract of man. In: The Function of the Ureter and Renal Pelvis. Philadelphia: PA; WB Saunders; 1957.

20. Pearle MS, Pierce HL, Miller GL, et al. Optimal method of urgent decompression of the collecting system for obstruction and infection due to ureteral calculi. J Urol 1998;160:1260-4. hittp://dx.doi.org/10.1016/ S0022-5347(01)62511-4

Correspondence: Dr. Barak Rosenzweig, Department of Urology, The Chaim Sheba Medical Center, Tel Hashomer, Ramat-Gan, Sackler School of Medicine, Tel-Aviv University, Israel; barak22@gmail.com 